\title{
A USEFUL COVER-SLIP CONTAINER
}

BY

\author{
J. C. MONCKTON \\ From the Southern Group Laboratory, Park Hospital, London
}

(RECEIVED FOR PUBLICATION NOVEMBER 16, 1950)

Most laboratory workers have experienced occasional difficulty in removing a cover-slip from its box to the slide. A container has been devised which reduces such mishaps to a minimum.

The container consists of a "perspex" box, with a central horizontal slot in the front and in the back. Through this passes a glass microscope slide with a cover-slip $\left(\frac{7}{8}\right.$ in. sq. No. 1) fixed to the upper surface. Inside the box, resting on the slide, is a supply of clean cover-slips, and one is delivered through the front slot each time the slide is pushed backward and forwards.

The method of working is seen in the diagram (Fig. 1a), which shows the slide in the three positions required for its operation. The slide and cover-slip are shown as thick black horizontal lines. The thickness of the cover-slips is exaggerated to show the working more clearly.

\section{Construction}

$\mathrm{A}^{*}$ cover-slip is first mounted on a microscope slide with one edge $\frac{5}{8}$ in. from the end of the slide. Use the smallest possible amount of DPX or Canada balsam to avoid seepage beyond the edges of the cover-slip. Place in an incubator for 48 hours to dry with a small weight on the cover-slip.

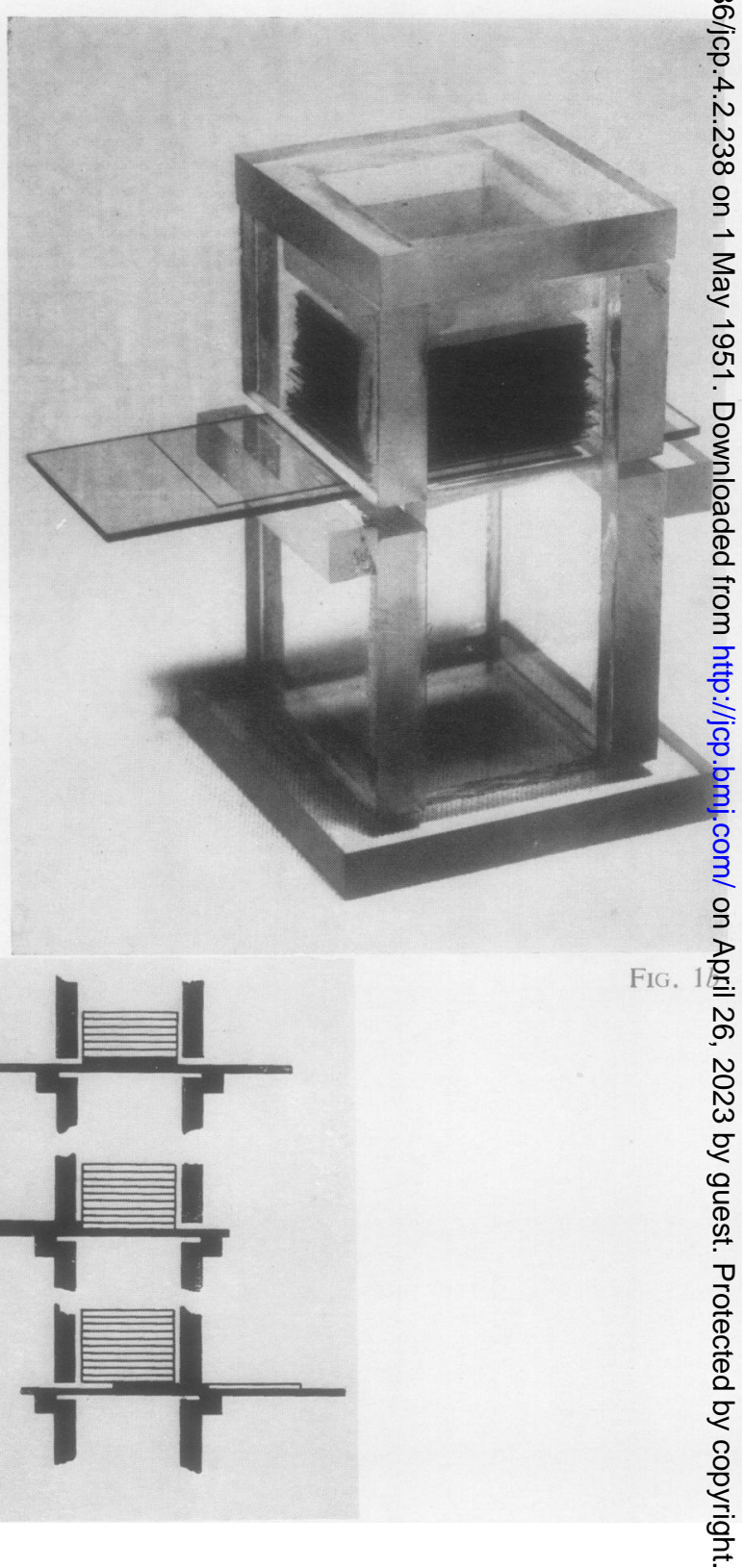


The box is made from "perspex" sheet $7 / 32$ in. thick. It is cut to the sizes required and the edges are smoothed.

The sizes of the sections are as follows:

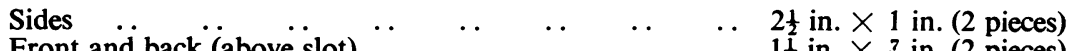

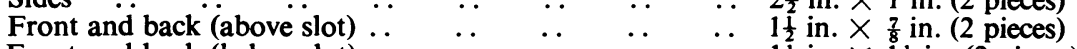

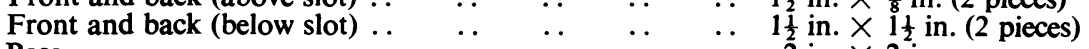

$$
\begin{aligned}
& \begin{array}{lcccccccc}
\text { Base } & . . & . . & . . & . . & . . & \ldots & \ldots & 2 \text { in. } \times 2 \text { in. } \\
\text { Lid (topside) } & \ldots & . & . & . & . & . & \ldots & 1 \frac{1}{2} \text { in. } \times 1 \frac{1}{2} \text { in. }
\end{array} \\
& \begin{array}{llllllll}
\text { Lid (underside) } & . & . . & . . & . & . & . & 1 \text { in. } \times 1 \text { in. }
\end{array}
\end{aligned}
$$

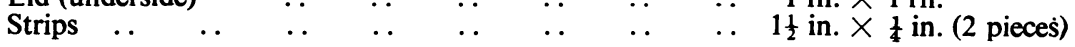

The parts are assembled to form the box with a central slot $\frac{1}{8}$ in. wide in the front and back as shown in Fig. $1 b$.

We used a little " perspex" dissolved in chloroform as cement. The removable lid has the smaller piece fixed to its underside to keep it in place.

The two small strips immediately below the slots are fitted last. Their purpose is to correct the width of the slots to the thickness of the moving slide and cover-slip. Their position is determined by placing the selected slide through the box with the affixed cover-slip emerging from each slot in turn and cementing a strip to the front and back of the box below the slide, so as to allow just enough room for the slide and cover-slip.

The container can be fixed to the bench with two small screws through the base if desired. This will enable one to manipulate the slide with one hand. 